

\title{
Collaborative Interactions for Medical e-Diagnosis
}

David Fuin, Eric Garcia, Hervé Guyennet, Jean-Christophe Lapayre

\section{To cite this version:}

David Fuin, Eric Garcia, Hervé Guyennet, Jean-Christophe Lapayre. Collaborative Interactions for Medical e-Diagnosis. HPCN, Int. Journal on High-Performance Computing and Networking, 2008, pp.189-197. hal-00561339

\section{HAL Id: hal-00561339 \\ https://hal.science/hal-00561339}

Submitted on 1 Feb 2011

HAL is a multi-disciplinary open access archive for the deposit and dissemination of scientific research documents, whether they are published or not. The documents may come from teaching and research institutions in France or abroad, or from public or private research centers.
L'archive ouverte pluridisciplinaire HAL, est destinée au dépôt et à la diffusion de documents scientifiques de niveau recherche, publiés ou non, émanant des établissements d'enseignement et de recherche français ou étrangers, des laboratoires publics ou privés. 


\title{
Collaborative Interactions for Medical e-Diagnosis
}

\author{
D. Fuin, E. Garcia, H. Guyennet and J-C. Lapayre* \\ Laboratoire d'Informatique de l'Université de Franche-Comté (LIFC) \\ 16, Rte de GRAY - 25030 BESANCON CEDEX - FRANCE \\ E-mail: [fuin,garcia,guyennet,lapayre]@lifc.univ-fcomte.fr \\ *corresponding author
}

\begin{abstract}
The Network and Distributed Systems Group within the University of Franche-Comte's computer research lab (LIFC) gained solid expertise on medical ediagnosis in the area of remote collaboration through continued research and findings. $\mathrm{TeNeCi}$ (Cooperative Teleneurology) is a European remote diagnosis project applied to neurology developed under the aegis of INTERREGIII. INTERREGIII is a European Community Initiative program aiming at supporting cross-border, transnational and interregional co-operation in both social and economic perspectives.

This paper has a dual objective: it first presents the improvements and contributions made to advance the TeNeCi project which is a research and development tool, and then it synthesizes our research work in collaborative medical e-diagnosis. The TeNeCi tool originality is to allow practitioners to act as if they were at the same diagnosis table, using a great panel of medical tools (images, software, ... ). Collaboration and awareness features are used to make TeNeCi more efficient than classical telemedicine software in terms of collaboration level.
\end{abstract}

Keywords: Tele-Diagnosis, Synchronous Collaboration, Asynchronous Collaboration, Collaboration Consistency, Sharing Consistency, Telepointer.

Reference to this paper should be made as follows: Fuin, D. Garcia, E. Guyennet H. and Lapayre J.-C. 'Collaborative Interactions for Medical e-diagnosis', Int. Journal of High Performance Computing and Networking (IJHPCN), A Special Issue on Recent Advances in Collaborative Internet Computing, 2005.

Biographical notes: David Fuin received his $\mathrm{PhD}$ in Computer Science from University of Franche-Comte. His current topics of interest are quality of Service, networks and distributed systems.

Eric Garcia is Assistant Professor at the University of Franche-Comté. He received a $\mathrm{PhD}$ in computer science in 2001. He is now working on collaborative work and network in the Network and Distributed Systems group at the LIFC, the research laboratory for computer science at the university. He is involved in two European research projects (Proteus/telemaintenance and TeNeCi/telemedecine).

Hervé Guyennet is Professor in Computer Science at the University of Franche-Comté. He is working on distributed systems: load balancing, collaborative work, distributed platform, multimedia, QoS. He is the head of the Network and Distributed Systems Group at the LIFC. He has been the scientific advisor for 12 theses. He is the FrancheComté representative of the ITEA European project Proteus/telemaintenance)

J.-Christophe Lapayre is Professor in Computer Science at the University of FrancheComté. He is member of the Network and Distributed Systems Group (LIFC). His research area includes Multimedia Collaborative Work. He is interested in applying distributed algorithms theories to manage concurrency accesses in collaborative work. $\mathrm{He}$ is the head of the InterregIII European project TeNeCi/Telemedecine. 
Figure 1: PACS

\section{INTRODUCTION}

Over the past 10 years, the concept of working remotely (teleworking) has been in rapid development. This phenomenon is due in large part to the parallel growth in high performance networks and processors. Teleworking is used in such various ways as distance learning, remote maintenance and even telemedicine. The Network and Distributed Systems Group at the LIFC (research laboratory for computer science at the university) has acquired a good experience on collaboration management for medical e-diagnosis. The TeNeCi (Cooperative Teleneurology) project (TeNeCi European Consortium, 2005) is a part of INTERREGIII program in collaboration with Swiss partners (Vaud University Hospital at Lausanne, and EPFL Lausanne).

Telemedicine is generally used in a nonacute setting for patient monitoring or education and has only recently been introduced into emergency care. Telemedicine can be defined as the use of telecommunication technologies to provide medical information and services. It is the process by which electronic, visual and audio communications are used to support practitioners at remote sites with diagnosis and consultation procedures, such as remote clinical examinations and medical image transfers.

For this kind of application, graphic interface and additional tools must facilitate actors' capacity to disregard distance and time in order to reconstitute a virtual examination room. Software and network architecture have to be optimal to improve interactivity and fault tolerance. Our aim is to obtain a secure environment to exchange medical data, diagnoses and opinions.

The second section of this paper presents the issue of medical e-diagnosis environment and we list the groupware functionalities required in order to obtain a collaborative application. In section 3, different levels of collaboration are defined and different technical solutions are described. These solutions involve the use of communication and concurrency algorithms that we have previously developed. These algorithms are briefly described. The last part of the paper presents tests and implementation of tele-neurology application. This application has been used to make a telediagnosis between France and Switzerland. The feedback of French and Swiss well-known experts in neurology (the real end users) involved in this test was very good.
Figure 2: Cephalic Hematoma

\section{MEDICAL E-DIAGNOSIS ENVIRONMENT}

\subsection{Collaborative Teleneurology}

The diagnosis process includes the clinical examination and also the analysis of several other associated sources of data (Sankaran and Bui, 2000). These include CT-Scan, MRI and ultrasound static and moving images, neurophysiological studies and biological examinations, such as blood tests. In addition to videoconference standard tools, it is essential to be able to handle perfectly the different medical devices, especially for the imaging.

Medical imaging is the main part of a tele-diagnosis software in a medical environment. Operations of archiving and visualization of medical images are very specific ( $\mathrm{Ti}$ wana and Ramesh, 2000). In order to help users to handle these images, professionals of medical world and medical devices manufacturers have developed the DICOM standard (Digital Imaging and Communications in Medicine).

\subsection{TeNeCi Developed Tools}

Integration of functionalities compatible with the medical environment requires a work in collaboration with medical staffs. Each tool that we have developed have been validated and tested in different hospitals. We present here the generic tools that can be reused in all telemedicine applications: the DICOM explorer and the DICOM viewer (Garcia et al., 2005).

The DICOM explorer allows physicians to search and download on their computer terminals images from different medical equipments (MRI, PET). The PACS (Picture Archiving and Communication System) makes it possible to perform image acquisitions on modalities (CT scan, MRI, PET), to archive produced images, to use the network and to consult produced images. Our DICOM explorer exploits these two last points to access the images stored on the PACS. After the data download stage, the use of the viewer (figure 1) becomes possible. This viewer is not only a simple image editor; it allows us to extract the image part of the DICOM file and to integrate specific 
amine multichannel biological signals;

- Groupware functionalities such as:

- The tele-pointer can indicate areas on images and it is equipped to take on different shapes according to the operations to be performed;

- The tele-annotations (text or drawing) enable inscriptions to be made on medical images;

- Tele-setting operations allow users to modify images and to broadcast each operation to session members. These operations can be zooms, rotations, treatments, contrast and brightness modifications;

- The observation tele-diagnosis tool allows several persons to fill out a questionnaire during the observation of patients' reactions. At the end of the observation, the application performs a comparison of different doctors' evaluations and gives a summary report;

- Application sharing allows several practitioners to act in real time on the same complex application. For example, TeNeCi allows a specific and complex form recognition mono-user software launched on a single computer to be shared (displayed and remotely driven) by all the practitioners involved in the e-diagnosis.

tools used in medicine such as data manipulation tools (contrast, brightness, zoom, scroll, geometric transformations, pattern recognition). For example, a modification of contrast and brightness settings may highlight particular lesions such as a cephalic hematoma with rightward shift of midline (figure 2).

\subsection{Groupware Functionalities}

We have seen in the previous part that specific medical features have to be integrated, but it is also essential to provide advanced groupware functionalities (Sankaran and Bui, 2000; Martino et al., 2003). These advanced groupware functionalities common to all types of collaborative applications (Hong et al., 1998) should allow practitioners to act as if they were in the same examination room. Our aim is to create a virtual diagnosis area.

Functionalities (figure 3) to perform an efficient telediagnosis in medicine are:

- A videoconference system, with motorized IPcam and memory position (one position patient, one position patient face, one position patient eyes);

- Medical tools: Several neurological viewers are included in the TeNeCi software such as a DICOM viewer and an EDF viewer. The Dicom viewer enables to examine medical imaging by changing contrast and brightness of an image, to make appear vascular cerebral events. The EDF viewer makes it possible to ex-

Different characteristics have to be specially considered in collaborative telemedicine, in particular those involving real-time transmission on the network. In this class, telepointer and tele-settings are essential for a collaborative diagnosis or a collaborative tele-staff. We paid a great attention to obtain light and hard real time tools. We have worked to minimize the treatments to be performed and the data to be sent on the network.

We can classify TeNeci tools in 3 classes; (1) essential : tele-pointer, tele-annotations and tele-settings which have to be very light and efficient and must work on a low bandwidth network, it is impossible to work without them; (2) very useful : sound, it is possible but difficult to work without it but it can be replaced by a phone if we have only very poor network resources; (3) useful : video and external application sharing, it is only essential for a remote examination or specific and complex manipulations but in this case we have to be sure of the network.

\section{COLLABORATION IN TELEMEDECINE}

\subsection{Collaboration Levels}

We can define several collaboration levels which can be characterized by a communication type and the addition of groupware tools such as the tele-pointer.

Table 1 shows the communication and action types. The left column represents a user with or without writing rights 
and the top line represents the aimed remote collaboration site. The initiator of the collaborative task can share an object with one or several sites that can have or not modification rights. This allows us to show different collaboration levels corresponding to one cell in Table 1.

We do not represent in this table in the left column the possibility to have $\mathrm{N}$ initiators because we only want to underline the communication and action aspect. $\mathrm{N}$ initiators are possible, but introduce awareness, properties on the work and replication problems that we do not want to treat in this paper. The first cell (Min Display) corresponds to the minimum level of collaboration in terms of communication and actions (we do not take into account in this table additional collaboration tools such as the telepointer). It can be, for example, a fixed screenshot display of an application between 2 users $(1 \mathrm{R} \rightarrow 1 \mathrm{R})$.

The second cell $(1 \mathrm{R} \rightarrow 1 \mathrm{RW}$ : Hand Taking) corresponds for example to a VNC session (RealVNC Company, 2005) between two participants and the cell 1R $\rightarrow$ NRW corresponds to the same VNC session but with several users sharing the control. In the first line, the initiator site is inactive, so the collaboration level is not at its maximum. The greatest collaboration level is reached when one user can act on an object or on an application and can share the control with several remote users.

For the collaboration to be the best as possible, it is also necessary to add some groupware tools such as the telepointer. For example, a document sharing application for a PowerPoint presentation (1RW $\rightarrow \mathrm{NR}$ at time $t$ ) would be more efficient if a tele-pointer could be displayed, making it possible to discuss more precisely on a curve.

\subsubsection{Technical solutions}

In medicine, applications are very specific and complex (3D reconstitution, CT Scan film mode). Our aim is to be able to integrate a maximum of medical applications in our platform without redeveloping them. A medical application designed to run on a single station, manipulated by a single user, should be integrated in order to make it the more collaborative as possible. There are several types of applications, and we will see different solutions to reach our aim. Each solution has advantages and drawbacks and we try to measure it using the classification presented in Figure 4. The latter figure presents the possible types of applications to integrate, the different technical solutions that can be used to reach a given communication
Figure 4: Cost of the Different Solutions

and action level (Table 1), and the possibility to add a collaborative tele-pointer to improve the collaboration. This classification gives several solutions (paths). Each solution can be analyzed to determine the best way to make collaborative an application. The result $(\mathrm{L}, \mathrm{D}, \mathrm{P})$ has to be analyzed, where: $\mathrm{L}$ is the Level of collaboration obtained using a certain path; $\mathrm{D}$ is the development time needed; $\mathrm{P}$ is the performance of the final shared application in terms of network and CPU use.

The first column represents the possible types of applications:

- OAE are Open Application Entities. In this case we can access the entire code of the application. Thus, it is possible to modify them and for example to replicate an application on several sites and to send only commands on the network to make it collaborative;

- HOAE are Half Open Application Entities. In this case, only a few functions are available to interpret some commands sent from remote sites;

- CAE are Closed Application Entities. In this case, there is no possibility to act at the application level to make it collaborative.

The second column represents the technical solutions that can be used to make applications collaborative. Usable solutions are function of the kind of application we choose to integrate (OAE, HOAE, and CAE). There are 3 main technical solutions:

- SC (Screenshot): in this case a screenshot of an application can be taken and sent periodically to one or several users. In this case, the maximum collaborative level that we can reach in term of communication and action is $1 \mathrm{RW} \rightarrow \mathrm{NR}$;

- VAE (Virtual Application Entity): this solution uses the previous one but it is also possible to capture some system events (mouse, keyboard) on remote sites and to apply them on the initiator site. This solution has to be coupled with a strict concurrency and consistency management algorithm (see next section);

- RAE (Replicated Application Entity): in this case, the application is launched on all sites; only commands are 
captured and broadcasted to everyone. It is also imperative to use consistency and concurrency management algorithms.

Only VAE and RAE allow us to reach the maximum level of collaboration (1RW $\rightarrow$ NRW), so they also allow us to reach all other levels. The addition of the tele-pointer increases the collaboration level but can add some development time. For a given collaboration level, there are several solutions (paths). Our classification allows us to highlight the advantages and drawbacks of each path. For example, in term of efficiency (network, CPU) the RAE solution is the best, but it is the worst in term of development time. If we want to integrate an existent complex closed application $(\mathrm{CAO})$, we have to use the SC or VAE solution; the choice must be motivated by the collaboration level we wish to obtain.

The use of our classification allows us to analyze and to choose the best technical solution to meet the collaboration application requirements. It can be used to create an efficient link between the design and the technical implementation, taking into account concrete features such as development time and performance.

\subsubsection{Collaboration Management}

In the situation 1RW $\rightarrow$ NRW, we obtain the highest level of collaboration. It is imperative in this case to define concurrency techniques (Park, 2004; Kumar et al., 2004; Guan and Lim, 2004). The LIFC (France) works on these problems since 1995. A notion of ownership on objects or applications has to be integrated. Traditionally, a user takes the control on an application and releases this control after its operations. In our case, it is more interesting to make the applications more flexible by allowing several persons to act in real time on the data. Multiple solutions are possible. A notion of ownership can be used, and we can reduce the granularity of objects. The entire application is not locked anymore, but a user can obtain the ownership on an object, an action or a part of the application (Garcia et al., 2001).

This introduces problems of concurrency, ownership, consistency, complementarity management. We have developed and designed different algorithms to manage these problems: ownership obtaining, concurrency in case of modification in nomad mode, fault tolerance (Garcia et al., 2005)... These algorithms (the Pilgrim and the Chameleon) are based on different communication principles (centralized, token... ) and allow us to obtain a robust and efficient management of the problems generated by the collaboration.

The Pilgrim is a token ring based protocol, which orders access on shared objects to allow the management of concurrency problems. But the main default of a token ring based protocol is to visit all sites including noproducers, therefore we have developed the Chameleon protocol, which allows the virtual topology to be reconfigured.
Pilgrim algorithm allows us to manage consistency of distributed shared memory. It uses the principle of property on shared objects. Some optimizations have allow us to implement a better version of this protocol on a token ring topology. This type of fixed topology limits its efficiency for collaborative applications. A complementary use of the Chameleon algorithm is an interesting alternative to this problem, it allows us to take advantage of robustness and performances of these two combined algorithms. For example, messages scheduling and property obtaining on contrast and brightness settings in the Dicom viewer are managed by the Chameleon and the Pilgrim.

\subsection{Interaction Modes}

Our collaborative tele-neurology application works with two complementary modes:

- Asynchronous mode: one person performs the diagnosis with the tools provided by the application. This mode can be composed of an image search stage to establish a diagnosis, followed by an emission stage to ask for an opinion, for example. This opinion can be given a few days after reception. For this mode, the interface must provide the necessary tools to create a package which must allow the expert to view images and final diagnosis and also to reconstruct how the diagnosis was posed (annotations, image settings, treatments);

- Synchronous mode: it uses the same tools allowing real-time collaboration among several people. This mode provides mechanisms to manage concurrent access to particular commands, e.g. contrast and zoom etc. It is composed of an image search stage, not in order to pose the diagnosis directly and to broadcast information to each person involved in the real-time collaborative diagnosis.

\subsubsection{Asynchronous Mode Needs}

This mode involves a search and download stage using our DICOM Explorer. After this stage, the doctor can perform a diagnosis by using the platform tools (annotations, drawing, settings modification).

The HCI (Human Computer Interface) provides a packing option to create an archive (package) which contains downloaded images, patient information, modifications performed on images, files (text, diagnosis, audio/video). This package can be stored for later examination and it can be sent to an expert or used to enrich a knowledge database.

In the case of emission, it is possible to specify the addresses. The message will be sent to the platform mailbox of this person who will open this package at a later stage. The opening process (unpacking) loads the file on the interface giving a precise view of the how the colleague posed the diagnosis. The expert contacted can display operations performed and results obtained and he can also work on the data. He gives his opinion by replying (packing and 
reply) to the original correspondent. Packages can be sent to several people.

\subsubsection{Synchronous Mode Needs}

Packing and unpacking operations are also used for realtime interactions directly after the session instigator has downloaded data from the PACS. In this case, receivers (users registered in the session and chosen by the instigator) obtain an automatic display of the entire file on their graphic interface. Each one could then act in real time (according to permission and priority policies in order to keep a good order in the collaboration). For this mode, we distinguish several types of functionalities:

- The videoconference tool ensures a human aspect is maintained during collaboration;

- The geographic and software topology representation makes it possible to geographically locate each person involved and to distinguish the communication architecture used. This feature is very useful in visualizing the access rights and activity of people involved in posing the diagnosis (Garcia et al., 2005);

- The DICOM Explorer tool used only by the instigator in order to search and download images;

- The file transfer tool allows users to broadcast a video file of the patient during the collaborative diagnosis;

- Groupware functionalities (section 2.3) are used.

For this particular mode, HCI and permissions are very important in order to manage collaborative work without breaking the collaborative feeling of the virtual examination room. This requires the implementation of a visible hand taking system on different operations in order to determine precisely the initiator of an action. We use a marker for each functionality, which allows a physician to modify the brightness while another, draws on the image for example (the entire platform is not locked for one person). The TeNeCi platform has to control group members while avoiding the impairment of each member's initiative. This can be difficult, however, the videoconference tool can help to overcome this obstacle allowing sessions to be organized as in a classical meeting where participants are physically present in the same place.

\subsection{Telemedicine Scenario}

We describe in this section a scenario of tele-diagnosis using asynchronous and synchronous mode. In this scenario, 3 neurologists are involved, two in France, and one in Switzerland.

(1) The first doctor downloads medical images of its PACS (medical imagery server of the French hospital), he annotates these images, adds a video of the patient examination. Then, he creates a diagnosis package and sends it to his French colleague (Asynchronous Mode);
(2) The second doctor opens this package few hours later. He recovers the diagnosis reasoning of its colleague. He adds annotations and drawings on scanner images and replies to the initiator (Asynchronous Mode);

(3) On the first French side, the diagnosis is uncertain, it still disagreements and perhaps misunderstanding with the second French side, so a real time meeting is programmed between the 2 parts and a third neutral part in Swiss (Synchronous Mode);

(4) The 3 participants connect them in Synchronous Mode. They use the videoconference tool to see the patient or to speak with the other practitioners. Groupware tools (tele-pointer, drawing, settings modifications) are usable on MRI images. It is also possible to use a collaborative $3 \mathrm{D}$ reconstitution application (using our application sharing tool) to evaluate the size of a tumor. The Synchronous or Real-Time mode allows us to remove misunderstandings thanks to videoconferencing and to groupware tools. This is not possible (or with an enormous number of exchanges) in asynchronous mode. So, even if this first mode is the more used in the majority of the cases, the synchronous mode (the more complex to deploy) is essential.

\section{TESTS AND IMPLEMENTATION}

\subsection{Software architecture}

The TeNeCi application is composed of several modules presented in figure 5 . The TeNeCi server is the core and contains:

- The repository (name, login, password, access control, picture, options ....) of authorized persons;

- The connection server registers connected persons and maintains a list of IP and port numbers;

- The TeNeCi mail server contains the list of received packages for each user. These archives can be stored on the server as for POP3 protocol in case of ordinary e-mail;

- The observer stores all performed actions;

- The coordinator which is a kind of proxy.

TeNeCi applications provide the different tools we have presented after an authentication/inscription stage through the TeNeCi server (figure 5) in order to be enrolled in a collaborative diagnosis.

Different communication modes (centralized, token based) between applications are usable, according to different parameters. These parameters; advantages and disadvantages of different communication algorithms are detailed in (Garcia et al., 2005).

This communication layer lies on a secured network, indeed, during users inscriptions; an IPSec VPN is created in 
Figure 5: TeNeCi Architecture

Figure 6: Optimal Pilgrim Size

order to avoid the transmission of unencrypted data. The registration stage is also secured. All data stored on computers (packages) are also protected. The network architecture of the TeNeCi platform (Switzerland-France) offers a real $8 \mathrm{Mb} / \mathrm{s}$ throughput (tests performed in 2005). This bandwidth makes it possible to use each of the platform tools and guarantees the performance required for the synchronous mode.

\subsection{Tests}

\subsubsection{Tests on Sharing Management Protocols}

It seems important to indicate our implementation performance according to Pilgrim size (section 3.1.2). This size depends on to the collaborative application type. This protocol is used for consistency management of discrete medias: for example visio conference tool do not use this protocol.

In Figure 6, we see that the optimal Pilgrim size is between 5000 and 10000 bytes on a $10 \mathrm{Mb} / \mathrm{s}$ network. But for 5 collaborative members, performance does not decrease proportionally to Pilgrim size. Pilgrim implementation is efficient for all types of discrete media applications, where the size of objects is not too great and no synchronization between sites is needed. We can conclude that the Pilgrim is adapted to TeNeCi requirements to manage the concurrency on discrete tools such as the Dicom viewer, the tele-settings tools...

Many performance of the Chameleon are produced in (Garcia et al., 2005). This algorithm is especially efficient in fluctuating environment (in term of number of users, network load, interaction speed, ... ): e-diagnosis with pa- tient, practitioners (entries and exits of members during execution).

\subsubsection{Test on Teleneurology Software}

Synchronous and asynchronous modes are implemented, as well as image setting functions, packing and unpacking operations and transfer tools. Application sharing has been tested with Open and Closed Application Entities such as respectively the Shared Dicom Viewer and a 3D reconstruction tool.

The DICOM tool (Garcia et al., 2005) implemented using Java, uses JDCM (JavaDicom) API and is compliant with the DICOM 3.0 norm. It includes an API to access implemented DICOM services (verification, storage and Query/Retrieve services). In order to ensure the compatibility between DICOM modalities and our explorer, all transfer syntaxes described in the DICOM 3.0 standard have been implemented (Big Endian, Little Endian). Finally, trials have been carried out at the regional hospital (University Hospital Besançon, France) in order to test communication and compatibility between DICOM devices used in the project and our DICOM tools. Good results have been reported.

A preliminary demonstration of the TeNeCi platform was carried out in May 2005 in the presence of French and Swiss doctors. This application runs on 2,5 GHz work stations equipped with 2 output video cards (GE Force FX 5200 ) in order to be able to use 2 screens to display the TeNeCi application (figure 3).

These tests, performed on a $8 \mathrm{Mb} / \mathrm{s}$ network between Besançon (France) and Lausanne (Switzerland) hospitals, have given very good results. The average latency varies between $100 \mathrm{~ms}$ and $500 \mathrm{~ms}$ depending on the functionality (100 ms for the tele-pointer to $500 \mathrm{~ms}$ for the video).

The bandwidth used depends on the number of users and the type of tools: for 2 users, $664 \mathrm{~Kb} / \mathrm{s}$ are used for a complete session with medium video quality $(2 * 56 \mathrm{~KB} / \mathrm{s}$ for the sound, $2^{*} 256 \mathrm{~KB} / \mathrm{s}$ for the video, $2^{*} 20 \mathrm{~Kb} / \mathrm{s}$ for the commands of the tele-pointer, tele-settings and teleannotations). We do not speak here of the application sharing tools, since it can consume in the worst case a very great part of the available bandwidth depending on the size and the quality required by each specific application. For example it can use $10 \mathrm{~Kb} / \mathrm{s}$ if the application is replicated (in this case only commands are transmitted) to $10 \mathrm{Mb} / \mathrm{s}$ for an application present on only one computer and requiring high quality images (in this case commands and images have to be transmitted)

When the number of users increases, several mechanisms aimed to reduce the used bandwidth and to allow the scalability. For the video, an adaptation mechanism reduces the quality and the size of the images dynamically and we can also send only the video of active users. Concerning the sound, we use a mechanism of multiplexing which merges several audio flows in single one. For example, with four users without this mechanism, the total bandwidth used for the sound is $672 \mathrm{~Kb} / \mathrm{s}\left(12^{*} 56\right)$ and with our mecha- 
Figure 7: Mobility in TeNeCi

nism, it is reduced to $336 \mathrm{~Kb} / \mathrm{s}(3 * 56+3 * 56)$. Usually in such applications 6 users is a maximum so technically we can handle the amount of communication on today networks.

Two well-known neurologists, Pr T. Moulin (France) and G. Devuyst (Switzerland), have tested this software and have made good comments, especially on the video quality of the motorized camera and on the fluidity of the telepointer and tele-settings. The collaborative aspect (everybody can act in real-time and at the same time on each tool) has been qualified of innovative. The only negative feedback was on the HCI that we are redeveloping by integrating 3D functionalities and simplifying the navigation.

\section{CONCLUSIONS AND FURTHER WORK}

In this paper, we have presented new usage modality of collaboration in e-diagnosis applications. These new functionalities was developed in order to conform to the new usage involved by telemedicine and e-health.

$\mathrm{TeNeCi}$ is now in test between the neurology departments of Besançon hospital (CHUB) and Lausanne vaudois hospital (CHUV). End users feedbacks are very good and a startup will be created to distribute this software.

Presently we focus our research on mobile e-diagnosis (figure 7) and especially on the different types of adaptation needed for collaborative work over wireless network, and with mobile terminals.

\section{REFERENCES}

Garcia, E. Droz-Bartholet L. and Haberbusch J.-L.(2005) 'A Collaborative Tele-Neurology for Remote Diagnosis, Conformance Statement for the Dicom Explorer, Technical Report RT2005-01, LIFC - University of Franche Comte, January.

Garcia, E. Guyennet H. and Lapayre J-C. (2005) 'Group Partitionning over CORBA for Cooperative Work', Journal of Cluster Computing, To appear 2005.
Garcia, E. Henriet J. and Lapayre J.-C. (2005) 'Study of an Optimistic Protocol for Concurrency Management in CSCW', In Procs. of the 9th IEEE Int. Conf. on CSCW in Design, LNCS, May, Coventry, UK, pp.128-133.

Garcia, E. Lapayre, J.-C. Sureswaran R. and Tharmaraj K. (2001) 'Centralized or Distributed Algorithm for Concurrency Management in Multimedia Conferencing Systems', In Procs of the Asia Pacific Advanced Network international conference, APAN 2001, August, Penang, Malaysia, pp.108-119.

Guan S. U. and Lim S. S. (2004) 'Modeling Adapatable Multimedia and Self-Modifying Protocol Execution', Future Generation Computer Systems, vol. 720, No. 1, pp.123-143.

Hong, JW. Shin, YM. Kim MS. and Shu YH.(1998) 'Design and Implementation of a Distributed Multimedia Collaborative Environment', Special Issue on Multimedia Collaborative Environments of Cluster Computing: Networks Software Tools and Applications, Baltzer Science, Fall.

Kumar S. and Munoz A. and Doganer T. (2004) 'Performance Comparison of Memory-Sharing Schemes for Internet Switching Architecture', Procs. of IEEE, 3rd International Conference on Networking, March, Vol. 1, pp.160-163.

Martino, R. L. Kempner K. M. and Govern F. S. (2003) 'A Collaborative Telemedicine Environment for the Ireland - Northern Ireland - National Cancer Institute International Partnership in Cancer Care', Procs of the 25th Annual Conference of the IEEE EMBS, September, Mexico, Vol. 5, pp.1350-1353.

Park G. L. (2004) 'Performance Evaluation of a List Scheduling Algorithm in Distributed Memory Multiprocessor Systems', Future Generation Computer Systems, Vol. 20, No. 2, pp.249-256.

RealVNC Company(2005) 'http://www.realvnc.com', 2002-2005, consulted in 2005.

Sankaran S. and Bui T. (2000) 'A Web-Based Correctional Telemedicine System with Distributed Expertise', Procs of the 33rd Hawaii International Conference on System Sciences, January 4-7, Maui, Vol. 5, pp.5019(10).

TeNeCi European Consortium INTERREGIII (2005) 'http://www.teneci.net'.

Tiwana A. and Ramesh B. (2000) 'From Intuition to Institution: Supporting Collaborative Diagnosis in Telemedicine Teams', Procs of the 33rd Hawaii International Conference on System Sciences, January 4-7, Maui, Vol. 5, pp.5018(10). 